Instituto Internacional de Investigación y Desarrollo Tecnológico Educativo INDTEC, C.A.

DOI: https://doi.org/10.29394/scientific.issn.2542-2987.2016.1.2.1.7-27

OAI-PMH: http://www.indteca.com/ojs/index.php/Revista Scientific/oai

\title{
Rasgos Convergentes y Divergentes entre las Escuelas Convencionales y las Escuelas Bolivarianas de Venezuela
}

\author{
Autora: Ana Gregoria Rivas Ruz \\ Universidad Nacional Experimental "Rafael María Baralt", UNERMB \\ rivasruz@hotmail.com \\ Mérida, Venezuela
}

\section{Resumen}

Al intentar explorar lo que representan los rasgos convergentes y divergentes en el marco de la reforma educativa basada en Diseño Curricular Bolivariana, encontramos que en Venezuela funcionan actualmente dos modelos de escuela: las escuelas convencionales y las escuelas bolivarianas. El objetivo de la investigación es realizar un estudio comparativo sobre los rasgos convergentes y divergentes que existen entre las escuelas bolivarianas y escuelas convencionales, estableciendo semejanzas y diferencias entre ambas modalidades en las instituciones educativas. Se sustentó en los Modelos Pedagógicos (Flórez, 2008) y el Diseño Curricular Bolivariano (2007). Metodológicamente la investigación es de naturaleza documental comparativa, apoyada en Hurtado de Barrera (2010), Universidad Pedagógica Experimental Libertador (2011), Sánchez (2000) y Pasek y Matos (2006). Como resultado se obtuvo que las escuelas convencionales y las bolivarianas, por una parte, confluyan los contenidos de enseñanza aprendizaje que dicta el currículo bolivariano, y los métodos de enseñanza por proyectos. Por la otra, divergen en el proceso de enseñanza, la atención integral a la comunidad y valoración de los saberes. Se concluye que hay diferencias en la formación de los estudiantes de acuerdo al método impartido, al tiempo de permanencia en la escuela y a su contacto, mayor o menor, directo o no, con la comunidad.

Palabras clave: escuelas convencionales; escuelas bolivarianas; enseñanza; aprendizaje; curriculum. 


\title{
Traits Convergent and Divergent Between Conventional and Schools of Venezuela Bolivarian Schools
}

\begin{abstract}
When trying to explore what they represent convergent and divergent traits in the framework of educational reform based on curriculum design Bolivarian Venezuela found that in two model school currently work: conventional schools and Bolivarian schools. The aim of the research is a comparative study of the convergent and divergent traits between the Bolivarian schools and mainstream schools, establishing similarities and differences between the two modalities in educational institutions. It was based on pedagogical models (Flórez, 2008) and the Bolivarian Curriculum Design (2007). Methodologically research is documentary in nature - comparative, leaning against Barrera Hurtado (2010), Pedagogical University Experimental Libertador (2011), Sánchez (2000) and Pasek and Matos (2006). As a result was obtained that conventional schools and bolivarianas one hand, converge the contents of learning which dictates the Bolivarian curriculum and teaching methods project. On the other, diverge in the teaching process, comprehensive care to the community and assessment of knowledge. It is concluded that there are differences in the formation of students according to the given method, the time spent at school and their contact, more or less, directly or not, with the community.
\end{abstract}

Keywords: conventional schools; bolivarian schools; teaching; learning; curriculum.

Date Received: 23-08-2016

Date Acceptance: 08-09-2016 


\section{Introducción}

No es fácil intentar reflexionar sobre un tema tan polémico pero interesante referido a la comparación de los rasgos convergentes y divergentes entre las escuelas convencionales y las escuelas bolivarianas de Venezuela, sobre todo cuando se pretende encontrar alternativas y herramientas que permitan equilibrar el justo medio en el proceso enseñanzaaprendizaje. En los últimos quince años, el sistema educativo venezolano ha realizado transformaciones curriculares en sus las líneas de acción, entendiéndose que la educación ostenta un papel preponderante en la formación integral del individuo y desarrollo del país que se quiere configurar. Fue así que surgió una propuesta educativa y proyecto bandera presidencial, denominado escuelas bolivarianas, para atender a los estudiantes de manera integrada.

Ahora bien, se ha detectado que este proyecto resulta divorciado de la realidad de muchas escuelas las cuales en el entorno de su proceso enseñanza- aprendizaje se denominan convencionales, y debido a las múltiples situaciones y condiciones de infraestructura, funcionan con unas determinadas características tales como: están distribuidas en una sola jornada de 5 horas diarias y 25 horas semanales. En éstas, el proceso enseñanza aprendizaje gira en forma interdisciplinaria en distintas áreas del currículo, en un contexto curricular de forma no integral. Por otro lado, encontramos que existen otras instituciones denominadas escuelas bolivarianas, que para cumplir con el objetivo del proyecto, debe tener dos jornadas de trabajo (Diseño Curricular Bolivariano, 2007) y funcionan, en una jornada de 8 horas diarias y 40 semanales, donde el estudiante desayuna, almuerza y merienda; pero además, realiza actividades académicas y complementarias de diversas índole.

Considerando, entonces, que ambos modelos de escuela obedecen al Diseño Curricular Bolivariano (2007), ofrecen el mismo contenido curricular, 
pero distribuido en distintas jornadas, surge la necesidad de visualizar ambas propuestas y realizar este estudio la cual consiste establecer los rasgos entre ambas escuelas y precisar las semejanzas y diferencias resaltadas en sus rasgos convergentes y divergentes. Teóricamente se sustentó en los Modelos Pedagógicos (Flórez, 2008) y en el Diseño Curricular Bolivariano (2007).

La investigación se realizó a través un estudio de naturaleza documental con un nivel comparativo, lo que permitió apoyo en las investigaciones de autores reconocidos tales como Hurtado de Barrera (2010), Universidad Pedagógica Experimental Libertador (2011), Sánchez (2000) y Pasek y Matos (2006). Este estudio contiene una introducción, las bases teóricas, la metodología y los resultados, terminando con las conclusiones.

\section{Bases Teóricas.}

Modelos Pedagógicos.

Entendemos por modelo pedagógico al conjunto de interrelaciones que permiten representar el acto de enseñar en sus diferentes dimensiones (psicológicas, sociológicas y antropológicas) y es a través de ellos, que la pedagogía ha incluido teóricamente diversos arquetipos que han transformado la educación y sus prácticas pedagógicas. En criterio de Rodríguez (1996), podemos sintetizar que dichos modelos son procesos de replanteamiento y de reconstrucción de todas las teorías y los paradigmas que sustentaran el paradigma educativo en función de las relaciones que predominan en el proceso de enseñanza - aprendizaje.

Por otra parte, Flórez (2008) expresa que "un modelo es la imagen o representación del conjunto de relaciones que definen un fenómeno, con miras a su mejor entendimiento" (pág. 160). En virtud de este criterio, se infiere que un modelo pedagógico es necesario para establecer que el análisis del fenómeno en estudio y no es únicamente un proceso analítico donde el todo es examinado en sus partes, sino también como un proceso de integración de 
relaciones. El sentido de parámetros pedagógicos es en su concepto, el trasfondo de explicaciones acerca de una concepción del ser humano, pues en sí mismos son un objeto interesante de estudio histórico para los científicos sociales, y en general responden al menos a los siguientes cinco fundamentos: 1.- El ideal de la persona bien educada que se pretende formar; a través de qué o con cuales estrategias metodológicas; 2.- Con que contenidos y experiencias educativas concretas; 3.- A qué ritmo o niveles debe llevarse el proceso formativo; 4.- Quién dirige este proceso pedagógico y 5.- En quien se centra.

En relación a lo expresado, Baptista y Flores (1983), manifiestan:

que los parámetros que se interrelacionan para el análisis de un modelo pedagógico están enmarcados en las metas educativas, los contenidos instruccionales, el estilo de relación entre docente - estudiante, los métodos de enseñanza, los conceptos básicos de desarrollo y el tipo de institución educativa (pág. 12).

En concordancia con las nociones enunciadas, estos modelos se clasifican en:

Modelo Pedagógico Tradicional. En referencia a los aportes expuestos por Cafux (1996), el modelo tradicional influyó notablemente en los procesos de enseñanza y en los sistemas educativos, este enfoque se originó en la escolástica, filosofía propia de la iglesia católica que imperó desde los siglos IX hasta el siglo XV, en donde el fin primordial de la educación estuvo dirigido a la recuperación del pensamiento clásico como resultado del renacimiento. A este modelo se la ha calificado de enciclopedista, a lo cual el autor refiere:

El contenido de la enseñanza consiste en un conjunto de conocimientos y valores sociales acumulados por las generaciones adultas que se transmiten a los alumnos como verdades acabadas; generalmente, estos contenidos están disociados de la experiencia de los alumnos y de las realidades sociales (pág. 15). 
Al respeto Flores (2006) sintetiza que "el modelo básico de aprendizaje es el academicista y los estudiantes son básicamente receptores".

En coincidencia con la anterior apreciación Canfux (1996) afirma:

El profesor, generalmente exige del alumno la memorización de la información que narra y expone, refiriéndose a la realidad como algo estático y detenido; en ocasiones la disertación es completamente ajena a la experiencia existencial de los alumnos y los contenidos se ofrecen como segmentos de la realidad, desvinculados de su totalidad (pág. 19).

Modelo Pedagógico Conductista. Al igual que el modelo pedagógico tradicional, el modelo conductista considera que la función de la escuela es la de transmitir saberes aceptados socialmente. Según este modelo, el aprendizaje es el resultado de los cambios más o menos permanentes de conducta y en consecuencia, el aprendizaje es modificado por las condiciones del medio ambiente. Al respecto, Flores (2008), expresa que "este modelo se desarrolló bajo la mira del moldeamiento meticuloso de la conducta "Productiva" de los individuos" (pág. 162). El modelo ha sido calificado de positivista en el sentido en que se toma como objeto del aprendizaje el análisis de la conducta bajo condiciones precisas de observación, operacionalización, medición y control. Según este autor (pág. 163), podemos resumir que el método es básicamente el de la fijación y control de los objetivos "instruccionales" formulados con precisión y reforzados con los fundamentos teóricos del conductismo. El aprendizaje es originado en una triple relación de contingencia entre un estímulo antecedente, la conducta y un estímulo consecuente. De acuerdo con el modelo conductista la meta del proceso educativo es el moldeamiento de las conductas que se consideran adecuadas y técnicamente productivas con relación a los parámetros sociales establecidos donde el maestro cumple la función de diseñador de situaciones de aprendizaje y en las cuales tanto los estímulos como los reforzadores se programan para obtener como resultado las conductas planificadas. Se 
enseña para el logro de objetivos de aprendizaje a nivel de competencias operacionalmente definidas, y se diseñan de modo que a través de la evaluación pueda medirse el nivel de los mismos. Por tanto, el foco de proceso de enseñanza es el aprendizaje.

Modelo Pedagógico Progresista. Está fundamentado en las ideas filosóficas que plantea el pragmatismo, básicamente las ideas pedagógicas progresistas se hacen evidentes en las propuestas de una transformación total del sistema escolar, convirtiendo al estudiante en el centro del mismo, alrededor de quien gira los procesos institucionales. Por su parte, De Zubiria (1994) considera que "la escuela nueva rompe con el paradigma. En su lugar, la nueva escuela defenderá, la acción como condición y garantía del aprendizaje" (pág. 100). Este autor sintetiza los siguientes postulados básicos que la escuela debe activar: El fin de la escuela no puede estar limitado al aprendizaje, la escuela debe preparar para la vida; los contenidos educativos deben organizarse partiendo de lo simple y concreto hacia lo complejo y abstracto; al considerar al niño como artesano de su propio conocimiento, el activismo da primacía al sujeto y a su experimentación; los recursos didácticos serán entendidos como útiles de la infancia que al permitir la manipulación y la experimentación contribuirán a educar los sentidos, garantizando el aprendizaje y el desarrollo de las capacidades intelectuales.

Para este modelo pedagógico, cada experiencia social es esencialmente educativa. Por lo tanto, la escuela es una institución social que debe concentrarse en los más efectivos medios para ofrecer al estudiante desde sus primeros años de escolaridad los recursos necesarios para cultivar la herencia cultural y desarrollar sus facultades que le permitan lograr fines sociales.

Modelo Pedagógico Cognoscitivista. El modelo pedagógico cognoscitivista, se sustenta en las ideas de la psicología genética de Jean Piaget, a lo cual Mones (1994) expone que "Esta corriente pedagógica es una 
variante de la escuela nueva y del progresismo pedagógico" (p.76). Al respecto, De Zubiria (1994) estima que "este modelo se podría llamar propiamente una teoría del conocimiento y no del aprendizaje ni de la enseñanza, considerando que los seres humanos utilizan procesos cognitivos que son diferentes en los niños y en los adultos" (pág. 116). En este aspecto, el carácter activo del sujeto en sus procesos de conocimiento y de desarrollo cognitivo parten de las aplicaciones de Piaget, desde tres puntos a saber:

- Como un elemento teórico que ofrece instrumentos muy definidos para evaluar y establecer los niveles de desarrollo cognitivo y moral de los individuos.

- Como una herramienta útil en el planeamiento de programas educativos que permite la organización del contenido curricular de acuerdo con los niveles de desarrollo alcanzados por los niños y en la clarificación de algunos métodos de enseñanza tales como el aprendizaje por descubrimiento.

Bajo estos aspectos, el aporte significativo de las ideas de Piaget, ha sido su teoría de desarrollo moral, relevante en el desarrollo de programas tendientes a desarrollar el juicio moral y los razonamientos que orientan las decisiones morales de los individuos. No obstante, Corral (1996) analiza que "la limitación de este modelo reside en no comprender suficientemente el carácter desarrollador y no solo facilitador del proceso de enseñanza, lo que reduce su rol de vida esencial para el desarrollo de sus procesos intelectuales" (pág. 107).

Modelo Pedagógico Crítico - Radical. En primer lugar, la pedagogía crítica se interesa en una crítica a las estructuras sociales que afectan la vida de la escuela, relacionadas con la cotidianidad escolar y la estructura del poder y por el desarrollo de habilidades de pensamiento crítico - reflexivo con el fin 
de transformar la sociedad. Según Peter McLaren (1996), la pedagogía crítica examina a las escuelas tanto en su medio histórico como en su medio social por ser parte de la hechura social y política que caracteriza a la sociedad dominante. Los docentes que aplican los enfoques de la pedagogía crítica coparticipan con sus estudiantes en la reflexión de sus propias creencias y juicios. De igual manera cuestionan críticamente los "textos" que se utilizan en los procesos de enseñanza (pág. 64).

En resumen, podemos señalar, que los modelos pedagógicos son clave trascendental en la relación docente- alumno, los cuales facilitan de acuerdo a sus peculiaridades identificar y elaborar proyectos educativos con vista a obtener nuevos niveles de eficiencia en sus postulados teóricos y metodológicos, los cuales dependerán de la misión y visión de las políticas y estrategias educativas implementadas en cada país, y de los rasgos particulares de cada institución. De allí la importancia de tener una visión más clara y coherente de lo que significan las escuelas convencionales y las escuelas bolivarianas, las cuales se describen a continuación.

\section{Escuelas Convencionales.}

Las escuelas convencionales se caracterizan por que los estudiantes reciben tres horas 45 minutos de contenidos académicos curriculares incluyendo en esa carga horaria destinadas a educación física artes plásticas y otras disciplinas, de tal forma los estudiantes reciben 19 horas 45 minutos semanales de contenidos curriculares, lo que determina que la jornada para los maestros de escuelas convencionales es de 5 horas diaria porque ahí está incluido el tiempo dedicado al receso o recreo que dedica el estudiante, al descanso y tiempo para merendar.

Por no poseer espacio de infraestructura o por abarcar una matrícula numerosa, las escuelas convencionales no pasan a gozar de los beneficios que otorga la escuela bolivariana. No obstante, el currículo nacional 
bolivariano del 2007 sostiene que el subsistema de educación primaria bolivariana de Venezuela contempla dos maneras de abordar la jornada escolar, una por laborar ocho horas académicas diarias, y otra de cinco horas académicas diarias.

Las escuelas convencionales como instituciones técnico administrativas, con dos turnos mañana y tarde garantizan también la escolaridad de los niños y niñas; cuyo objetivo es proporcionar la base esencial para la construcción de futuros aprendizajes y constituye la educación formal mínima obligatoria que deben cumplir los venezolanos según lo pauta la ley. En las escuelas convencionales la concepción de la enseñanza aprendizaje, se alimenta de los aportes de las diversas corrientes psicológicas asociadas a la psicología cognitiva entre las que se destaca:

a.- La Teoría genética de Jean Piaget. b.- Teoría del aprendizaje significativo de Ausubel. c.- Teoría socio - cultural de los procesos superiores de Vygotsky. Y d.- Otras teorías del desarrollo y el aprendizaje que a pesar de situarse en cuadros teóricos distintos, comparten el principio de la importancia de la actividad constructiva del alumno en el desarrollo de los aprendizajes escolares.

Es importante puntualizar que la escuela convencional gira en función de los siguientes aspectos: Por una parte, la atención y respeto a las desigualdades sociales y reconstrucción de conocimientos actitudes y pautas de conducta, (ya que el estudiante es el centro de la escuela y el maestro es orientador). Y por otra, un ambiente de aprendizaje constructivista y de acción comunicativa, originándose un triángulo educativo en la escuela convencional que corresponde a los contenidos el estudiante y el docente con interacción constructiva - comunicativa; estudiante - estudiante; estudiante - contenido, estudiante - docente. 


\section{Escuelas Bolivarianas.}

En este apartado, se señalan los puntos más resaltantes de las escuelas Bolivarianas, comenzando por sus orígenes: Se crean mediante Resolución Nº 179 del 15/09/1999, del Ministerio de Educación, Cultura y Deporte, y de conformidad con lo establecido en el artículo 30 del de decreto con rango y fuerza de Ley Orgánica de la Administración Central. En concordancia con los artículos $1^{\circ}, 6^{\circ}$ y $107^{\circ}$ de la Ley Orgánica de Educación tiene carácter experimental y abarca la población de preescolar, I y II etapa de educación básica.

La citada resolución en su artículo 4, establece que: el plan de estudio para las escuelas bolivarianas es el plan de estudio oficial que se rige en el país para los niveles de educación preescolar, primera y segunda etapa de educación básica, y certifica el uso de proyecto pedagógico de aula y otro similar, como un componente esencial para responder a la educación como un proceso participativo, centrado en los valores éticos de la democracia.

El concepto filosófico de la escuela Bolivariana es sutilmente humanista. O sea, un humanismo social que reclama el dialogo permanente del hombre con sus saberes y los de su comunidad, con la cultura de su sociedad y de todas las sociedades, permitiendo el crecimiento individual dentro del colectivo y con el colectivo.

Estas escuelas son consideradas espacios de participación y solidaridad de trabajo, de relaciones auténticas en las que participa la comunidad que brinda al docente, al estudiante y a los padres la posibilidad de acceder a diferentes fuentes del saber. En ese orden de ideas, se rigen por los siguientes principios:

- Una escuela transformadora de la sociedad donde se concrete e identifique la identidad nacional.

- Una escuela participativa y democrática. 
- Una escuela en, con y para la comunidad.

- Promover la justicia social y modelo de atención educativa integral.

- Permanente ejemplo de renovación pedagógica.

- Inclusión educativa que lucha a favor de los más desfavorecidos.

- Flexibilidad curricular enmarcada en el proyecto educativo nacional vinculado con el quehacer cotidiano, el trabajo y la conservación ambiental.

Misión de las Escuelas Bolivarianas. Brindar una educación integral a niños, niñas y adolescentes de práctica pedagógica, abierta, reflexiva, y constructiva estableciendo una relación amplia con la comunidad signada por la participación activa y protagónica, para un cambio efectivo en el sistema educativo acorde con los propósitos de construir un nuevo republicano.

Visión de las Escuelas Bolivarianas. Garantizar una educación democrática, participativa, protagónica, multiétnica y pluricultural que permita formar integralmente a niños, niñas y adolescentes, sin ningún tipo de discriminación; rescatando el ideario bolivariano en función de reivindicar el papel de la nación, que se reconoce en la historia.

\section{Objetivos de la Educación Bolivariana.}

El objetivo general de la educación bolivariana consiste en brindar acceso y permanencia a la población escolar y básica de las zonas urbanas marginales, rurales e indígenas, garantizando una educación integral de calidad. Este objetivo se desagrega en los siguientes objetivos específicos:

1. Ampliar la cobertura en los niveles de educación inicial y básica en las diferentes modalidades.

2. Mejorar la calidad de atención y condiciones físico ambientales de la educación a nivel nacional 
3. Incrementar el número de escuelas de horario integral en los diferentes niveles y modalidades.

4. Atender integralmente a niñas, niños y adolescentes, en edad escolar, en los niveles de inicial y básica.

5. Contribuir a superar la inequidad social.

La nueva escuela, la escuela bolivariana, entonces se propone los siguientes espacios:

- Espacio para la Formación Integral que promueva la justicia social.

- Espacio para las innovaciones pedagógicas en forma colectiva Escuela - Familia - comunidad.

- Espacio del quehacer comunitario que se vinculen con la localidad donde funcionan y a la cual sirven.

- Espacio de salud y vida orientado a la prevención, protección y defensa de la salud.

- Espacio de cultura y creatividad propicia la relación entre mente cuerpo y espíritu.

- Espacio para la Comunicación Alternativa que se oriente más allá del campo cognitivo.

- Espacio para las TIC a través de los centros Bolivarianos de Telemática e Informática.

- Espacio para la Paz orientada para el desarrollo de los valores de paz, tolerancia y solidaridad.

De acuerdo a lo planteado, podemos señalar que tanto las escuelas convencionales como las bolivarianas, no pueden limitar su proceso enseñanza- aprendizaje a procesos pedagógicos rígidos o encasillados a patrones inflexibles. Al contrario, el deber de las instituciones educativas es 
implementar y preparar con el apoyo de docentes y comunidad a los alumnos para y por la vida. De esta manera se debe partir de lo simple y concreto a lo complejo y abstracto para que el mismo alumno trate de moldear sus conocimientos y sea a su vez objeto de su propia experimentación, tratándo de garantizar el aprendizaje y desarrollo de las capacidades individuales en el proceso de construcción de los aspectos filosóficos, psicológicos, antropológicos y sociales, y, los docentes, impulsar el proceso de transformación individual y social de los alumnos ya sea en las escuelas convencionales o en las escuelas bolivarianas.

\section{Metodología.}

La investigación fue de naturaleza documental con un nivel comparativo. Como investigación comparativa, "está orientada a destacar la forma diferencial en la cual un fenómeno se manifiesta en contextos o grupos diferentes, sin establecer relaciones de causalidad." Hurtado de Barrera (2010) (p.106). Por su parte, la Universidad Pedagógica Experimental Libertador (2011), refiere como indagaciones documentales los estudios de desarrollo teórico, revisiones críticas del estado del conocimiento en el área específica de que se trate, estudios de educación comparada, entre otros. En ese sentido, la investigación se encuentra inserta en los estudios de desarrollo teórico, los cuales consisten en "la presentación de nuevas teorías, conceptualizaciones o modelos interpretativos originales del autor, a partir del análisis crítico de la información empírica y teorías existentes." (pág. 20). La originalidad del estudio se refleja, en este caso, en la construcción de criterios nuevos y/o adicionales que permiten comparar ambos tipos de escuela.

Para dar puntualmente respuesta al objetivo del estudio que fue determinar los rasgos convergentes y divergentes entre las escuelas convencionales y las escuelas bolivarianas de Venezuela, se integró en el procedimiento metodológico el proceso de comparación que propone Sánchez 
(2000), complementado con el proceso de la investigación analítica Hurtado de Barrera, (2010), considerando que, según ésta última, la comparación involucra un análisis previo. En consecuencia, se recorrieron los siguientes pasos:

1.- Se definió el propósito de la comparación, que consistió en determinar o concretar los rasgos convergentes y divergentes entre las escuelas convencionales y las escuelas bolivarianas de Venezuela.

2.- Se identificó las variables o eventos que definen el propósito; las características puntuales de las escuelas convencionales y las escuelas bolivarianas de Venezuela.

3.- Se definieron los eventos o variables, aspectos que conforman la fundamentación teórica del estudio, sustentados en los Modelos Pedagógicos Flórez, (1994) y Diseño Curricular Bolivariano (2007).

4.- Se elaboraron los criterios de análisis que permitieron dividir las variables o eventos en estudio en las dimensiones o aspectos.

5.- Se describieron los resultados y se elaboraron las conclusiones.

Los resultados en este caso, se entienden como algo provisional que podrá complementarse y reestructurarse sobre la base de estudios posteriores.

\section{Discusión de los Resultados.}

En este apartado se presentan los resultados de la comparación de los aspectos o rasgos que caracterizan las escuelas convencionales y las escuelas bolivarianas de Venezuela. En ambos casos se sigue el orden de los criterios establecidos a tal fin, los cuales son:

\subsection{Semejanzas entre escuelas convencionales y escuelas bolivarianas}

de Venezuela. Se encontró que ambos modelos de escuela se asemejan en 
la concepción de hombre, los contenidos enseñanza aprendizaje y el método de enseñanza aprendizaje basado en proyectos.

Concepción de hombre. En ambas modalidades escolares se pretende formar una concepción de hombre republicano, crítico y reflexivo. Esto significa que los y las estudiantes participen libremente en sus actividades, en su quehacer diario y que estén instruidos al enfrentar retos de participación, den sus propias opiniones y aportes, exploren sus conocimientos y que sean capaces de hace pensar en la toma de decisiones.

Contenidos enseñanza aprendizaje. Ambas escuelas siguen un mismo programa o contenidos programáticos. Es decir, se rigen bajo los contenidos del currículo bolivariano en las diferentes áreas de aprendizaje tal y como lo desglosa el mismo.

Método de enseñanza aprendizaje. Tanto en las escuelas bolivarianas como en las escuelas convencionales, el proceso de enseñanza aprendizaje se orienta básicamente con los proyectos de aprendizaje, los cuales lo realizan los docentes de acuerdo a las necesidades de los estudiantes.

\subsection{Diferencias entre escuelas convencionales y escuelas bolivarianas}

de Venezuela. Se encontró que ambos modelos de escuela se diferencian en:

Método de enseñanza aprendizaje. Aunque ambas modalidades de escuela aplican un proceso de enseñanza aprendizaje soportado en los proyectos de aprendizaje, en las escuelas bolivarianas dicho proceso incluye los espacios como complemento. Se entiende como espacios a los diferentes trayectos de trabajo que se realizan en cada una de las escuelas fuera del aula de clase y en la comunidad, mientras que escuelas convencionales los espacios de interacción educativa solo son representativos en el aula de clase.

En las escuelas bolivarianas, el hombre que se pretende formar es participativo puesto que hay una integración en cada espacio por lo que el 
estudiante está abierto a los cambios, entendiéndose espacios a las diferentes jornadas de trabajo que se realizan en las mismas, mientras que escuelas convencionales solo es en su aula de clase donde interactúan.

Por otra parte, en el método de enseñanza y aprendizaje utilizado en ambas escuelas es a través de proyectos de aprendizaje, el cual es una estrategia unánime de planificación escolar. Pero en el caso de escuelas bolivarianas, se integran los espacios para la paz, la salud y vida, para la cultura y creatividad, los tics, para la comunidad, para la formación integral, para las innovaciones pedagógicas, el quehacer comunitario, para la producción y la productividad.

Las Jornada de trabajo de escuelas convencionales se realiza en una jornada de 5 horas, desglosando 3 horas y media totalmente pedagógicas el resto en educación física y manualidades incluyendo receso, estas últimas cuando correspondan según horario establecido, en escuela bolivariana en una jornada de 8 horas diarias, las cuales se llevan a cabo de la siguiente manera 5 horas pedagógicas 2 recesos o descansos y 3 horas para áreas complementarias entre ellas se mencionan: Manos a la siembra, música, manualidades, teatro, computación entre otras.

Valoración de los saberes. En escuelas bolivarianas están presentes los saberes populares, libros vivientes de la comunidad, académicos entre otros. En las escuelas convencionales solo se evidencia el saber académico. En las escuelas bolivarianas la alimentación está distribuidas en: desayuno, almuerzo y merienda incluyente para todas las escuelas bolivarianas así como también salud con jornadas especiales para estas, de igual manera la cultura donde se integra los cultores de la localidad, los personajes que de una u otra manera hacen o hicieron historia llamados también libros vivientes, los espacios abiertos calificativo este para realizar las actividades de manos a la siembra, deporte, encuentro de saberes; lo cual a través de una actividad relevante hacen demostraciones de los saberes aprendidos en las 
instituciones tales como: cultura, gastronomía, manualidades, los cuales a su vez se subdividen en: música, poesía, declamación, canto ejecución de instrumentos y en teatro encuentra cabida los títeres y monólogos.

Objetivos de las escuelas bolivarianas y escuelas convencionales: la primera garantiza una educación integral de calidad y la segunda garantiza una educación de calidad observándose desde la óptica diferencial de la escuela bolivariana la integración del hombre desde las perspectivas en todos los espacios a abordar en el escenario de su formación.

Visión de escuelas. La visión de las escuelas bolivarianas es una educación demostrativa participativa, protagónica con rescate del ideario bolivariano, donde los libros son entregados a través de bibliotecas a todas las aulas de escuelas bolivarianas. En este sentido la visión de escuelas convencionales tiene su horizonte en formar un nuevo republicano, pero difiere la primera de la segunda a la educación integral, es decir participativa, que sea capaz de defender sus derechos en un colectivo, que tenga activación en la comunidad y su entorno, que produzca en su entorno.

Principios que las rigen. Ambas modalidades de escuela poseen como principios lo participativo y democrático, pero existen diferencias. Por ejemplo, la escuela convencional es participativa y democrática, fundamentalmente por su trabajo por proyectos, pero la escuela bolivariana es, además, trasformadora, porque permite una relación amplia de inclusión y flexibilidad con la comunidad, con el quehacer diario, promoviendo la justicia social.

Organización. Vinculados con todas estas concepciones las jornadas o momentos de clases se darán a través de la malla curricular denominada esta como la "ruta de trabajo" nombrando las distintas áreas de aprendizaje que se establece bajo un cuadro donde aparecen las áreas o contenidos grados y la cantidad de horas semanales distribuidas en áreas y grados. En las escuelas primarias convencionales con una jornada de 5 horas diarias 
además de esa distribución se desarrollan actividades de receso, refuerzan horas de aprendizaje de acuerdo a su necesidad mientras que en escuelas bolivarianas a esta cantidad de horas de agregan las complementarias recordando su trayecto de 8 horas diarias, que se fraccionan además de las 5 horas pedagógicas 3 horas de acuerdo a la necesidad pueden ser cultura manos a la siembra deporte y recreación, teatro, música, manualidades.

\section{Conclusiones.}

Es necesario tener presente que no existen métodos únicos para las escuelas convencionales y bolivarianas, sino una combinación de técnicas diseñadas en función de los objetivos, contenidos y sujetos del aprendizaje. De esta manera, se debe instruir periódicamente las prácticas educativas para empoderar a los educandos y educadores, familia y comunidad sobre los lineamientos que se pretenden incorporar, los que se quieren cuestionar y los que se quieren desincorporar del currículo escolar. El estudio comparativo realizado mediante una revisión documental sobre los rasgos convergentes y divergentes que existen entre las escuelas bolivarianas y escuelas convencionales, permitió establecer semejanzas y diferencias concretas entre ambas modalidades de escuela y así obtener una idea clara de las mismas. En la concepción del hombre republicano participativo y democrático; encontramos que se asemejan e igualmente en los contenidos enseñanza aprendizaje que dicta el Diseño Curricular Bolivariano (2007), y en el método de enseñanza aprendizaje por proyectos.

Los rasgos que las diferencian, se pueden agrupar bajo los siguientes criterios: la jornada de trabajo, el método de enseñanza aprendizaje por proyectos que incluye los espacios de encuentro, la valoración de los saberes, los objetivos que persiguen, la visión de escuelas, los principios que las rigen, y la organización o momentos de clase. 
Un punto a considerar es que las escuelas convencionales deberían contar con el mismo beneficio que las escuelas bolivarianas, ya que nuestra carta magna establece "una sola educación", de calidad e igualdad para todos. Cabe pensar, que tales diferencias pueden tener ciertas implicaciones educativas. En este sentido, se pueden señalar algunas implicaciones, tales como: la posible diferencia en la formación de los estudiantes debido al tiempo de permanencia en la escuela y a su contacto, mayor o menor, directo o no, con la comunidad. Esto les ofrece a los estudiantes de las escuelas bolivarianas más oportunidades de conocer su realidad.

Otro punto importante es indagar si realmente se está llevando a cabo la educación tal como está establecida para las escuelas bolivarianas, lo que involucra una educación de calidad. Dado que las escuelas bolivarianas cuentan con más beneficios, hay motivos para promover un solo tipo de jornada para el logro de la educación del hombre que se pretende formar.

\section{Referencias}

Flores, R. (2008). Hacia una pedagogía del conocimiento. Santafé de Bogotá: McGraw-Hill.

Hurtado, J. (2010). El proyecto de investigación. Comprensión holística de la metodología y la investigación. 6ta. edición. Caracas: Ediciones Quirón.

Pasek, E., y Matos, Y. (2006). Cinco paradigmas para abordar lo real. Revista Telos, Volumen 8, № 1, pp. 106-121. Recuperado de: http://virtual.urbe.edu/artectexto/TEL/TEL-018/TEL-018-007/texto.pdf

Sánchez, M. (2000). Desarrollo de habilidades del pensamiento. 10ma. reimpresión. México: Trillas. 
Ana Gregoria Rivas Ruz

e-mail: rivasruz@hotmail.com

Nacida en Timotes estado Mérida. Cursa estudios de

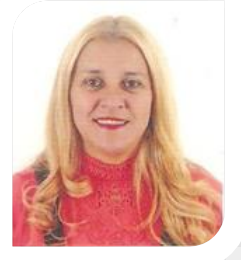
Doctorado en Educación, en la Universidad Rafael María Baralt Cabimas estado Zulia extensión Valera, especialista en Gerencia Educativa en la Universidad Santa María año (2001), licenciada en Educación mención Ciencias Sociales en la Universidad Nacional Abierta año (1999). Actualmente me desempeño como directora en el Liceo Bolivariano Francisco de Paula Andrade Timotes. Estado Mérida desde el año 2014 hasta la actualidad. Me desempeñe como supervisora municipal de educación en el Municipio Miranda del estado Mérida durante siete años consecutivos. Para el año 2005 me desempeñe como jefe municipal de educación en el mismo municipio. Contando con más de veinte cursos en el área de educación y un diplomado en supervisión educativa por el IPLAC ponente en congreso pedagógico municipal, jurado evaluador de tesis de gado en la Universidad Nacional Experimental de la Seguridad (UNES) y Universidad Nacional Rafael María Baralt. En la actualidad con una experiencia en aula de clase como docente en diferentes grados de primaria y educación media general con más de 19 años, así como también a nivel Universitario con dos años de experiencia.

El contenido de este manuscrito se difunde bajo una Licencia de Creative Commons ReconocimientoNoComercial-Compartirlgual 4.0 Internacional 